\title{
A COMPARISON OF DIFFERENT APPROXIMATE TWO-COMPONENT RELATIVISTIC THEORIES OF MANY-ELECTRON SYSTEMS: A CASE STUDY OF THE IONIZATION ENERGIES OF TWO-ELECTRON IONS
}

\author{
M. BARYSZ ${ }^{a}$, N. FLOCKE ${ }^{b}$ AND J. KARWOWSKI ${ }^{c}$ \\ ${ }^{a}$ Instytut Chemii, Uniwersytet Mikołaja Kopernika \\ Gagarina 7, 87-100 Toruń, Poland \\ ${ }^{b}$ Department of Chemistry and Physics, University of Florida \\ Gainesville, Florida 32611, USA \\ ${ }^{c}$ Instytut Fizyki, Uniwersytet Mikołaja Kopernika \\ Grudziądzka 5/7, 87-100 Toruń, Poland \\ (Received January 18, 2001; in final form May 9, 2001)
}

The corrections to the ionization energies of two-electron ions due to relativistic effects are studied by different two-component relativistic methods. In particular, the results obtained by the standard Pauli-Cowan-Griffin method and by two variants of the Douglas-Kroll-Hess method (the one based on the free-particle transformation and the one in which the transformation accounts for the nuclear potential) are compared with those calculated using the four-component Dirac-Fock method. Limits of applicability of each of these methods have been indicated. Results acceptable in the whole range of the nuclear charge (relativistic corrections accurate up to $4 \%$ for $Z \leq 85$ ) are given only by the Douglas-Kroll-Hess method which goes beyond the free-particle transformation. Each of the other two approaches either underestimates or overestimates the corrections due to relativistic effects.

PACS numbers: $31.15 .-\mathrm{p}, 31.30 . \mathrm{J}_{\mathrm{V}}$

\section{Introduction}

In spite of the very rapid and successful development of the "exact" four-component relativistic theory of many-electron systems [1-3], studies of two-component relativistic models are still timely and of relevance in quantum chemistry. During recent years methods based on the Douglas-Kroll transformed Dirac-Coulomb 
Hamiltonian [4-9] have received considerable attention because they have proved to be both accurate and easy to apply computationally [10-16].

The motivation for developing two-component relativistic approximations is their simplicity. Due to these methods, a substantial part of the relativistic effects may be taken into account by straightforward modifications of nonrelativistic computer codes. For this reason, the most common way of simultaneously including relativistic effects and electron correlation in molecular calculations are variations of the Pauli approximation. With the spin-orbit coupling neglected, this approximation is usually referred to as the Pauli-Cowan-Griffin (PCG) or the mass-velocity and Darwin (MVD) approximation [17]. The corresponding Hamiltonian is not bounded from below and is strongly singular [18]. Therefore this approximation has to be used with great care and, in principle, the operator describing the MVD correction should be used as the first-order perturbation.

A successful method to overcome the deficiencies of the MVD approximation has been developed by Hess [6] and is based on the Douglas-Kroll transformation [5]. The two-component Douglas-Kroll Hamiltonian is bounded from below and has no non-integrable singularities. Therefore it may be used in variational calculations employing any square-integrable trial function. The algebraic approximation introduced to the variational method based on the Douglas-Kroll transformed Hamiltonian by Hess $[6,10]$ has made it easy to apply computationally. The resulting formulation is referred to as the Douglas-Kroll-Hess (DKH) no-pair method. If the Douglas-Kroll transformation has been constructed using a free particle Hamiltonian [6] then, in this paper, the corresponding method is referred to as DKH0. If the Douglas-Kroll transformation depends on the external field potential [7] then the resulting method is denoted by DKH1. Usually, the two-electron terms (related to the Breit corrections) are not included into the Douglas-Kroll transformed Hamiltonian (see however [10, 19]). They are neither taken into account in this study. Their contribution to the total energy is similar to that due to the quantum electro-dynamics corrections [20], however they may be very important in determining the fine structure splittings in spectra [21]. Including the two-electron terms into the Douglas-Kroll transformation operator leads to a rather complicated formalism and, except for a general formulation [4] has never been implemented.

The routine calculation of relativistic corrections is still limited, mainly because most of the generally available software is based on non-relativistic formulations. Also the experience concerning the construction of suitable basis sets is rather limited. It has been recommended by Hess and co-workers [22-24] to construct appropriate basis sets by re-contracting the standard non-relativistic Gaussian basis sets by using the eigenvectors resulting from DKH Hartree-Fock (HF) calculations (rather than from the corresponding non-relativistic calculations). This strategy is due to the observation that the DKH bare-nucleus Hamiltonian emphasises a region closer to the nucleus than its non-relativistic counterpart. The effect of using basis sets optimised on the non-relativistic and on the relativistic levels is addressed in this study as well.

The aim of this investigation is to compare the relativistic corrections to the ionization energies of two-electron ions with the nuclear charge $Z$ ranging from 
$Z=2$ to $Z=86$ computed by different two-component methods. The corrections are calculated by using the standard PCG approximation and by the two variants of the Douglas-Kroll-Hess no-pair approach. The correlation effects are studied by using the coupled cluster (CCSD) method [25], in this case equivalent to the full configuration interaction (CI), and by the full CI method, modified to account for the relativistic effects. All calculations have been performed using the OpenMol system of programs [26, 27]. The results proved to be helpful in determining the limits of applicability of the investigated two-component approximations.

\section{Theory}

The spin free no-pair approximation based on the Douglas-Kroll transformation reduces the four-component relativistic one-electron Hamiltonian to a two-component form. The Dirac Hamiltonian in the momentum space is

$$
H_{\mathrm{D}}=c \boldsymbol{\alpha} \boldsymbol{p}+\beta m c^{2}+V
$$

where $c \approx 137.036$ a.u. is the velocity of light and $V(i)$ is the one-electron nuclear attraction operator, can be transformed into a block-diagonal form exact to the second-order in $V$

$$
H_{\mathrm{D}}=\beta E_{p}+\mathcal{E}_{1}-\beta\left(\mathcal{W} E_{p} \mathcal{W}+\frac{1}{2}\left\{\mathcal{W}^{2}, E_{p}\right\}\right)
$$

where \{\} means anticommutator. In the spin-free approximation

$$
E_{p}=c \sqrt{p^{2}+m^{2} c^{2}}
$$

and

$$
\begin{aligned}
& \mathcal{E}_{1}=A(V+\boldsymbol{R} V \boldsymbol{R}) A, \\
& A=\sqrt{\frac{E_{p}+m c^{2}}{2 E_{p}}}, \\
& \boldsymbol{R}=\frac{c \boldsymbol{p}}{E_{p}+m c^{2}} .
\end{aligned}
$$

The integral operator $\mathcal{W}$ is defined in terms of its kernel

$$
W\left(p, p^{\prime}\right)=A(p)\left[R(p)-R\left(p^{\prime}\right)\right] A\left(p^{\prime}\right) \frac{V\left(p, p^{\prime}\right)}{E_{p^{\prime}}+E_{p}},
$$

where $V\left(p, p^{\prime}\right)$ denotes the Fourier transform of the nuclear potential $V$. Usually the Douglas-Kroll transformation is limited to its one-electron part and the two-electron terms are left in their Coulomb form. If the spin-dependent terms are neglected (as it has been done in Eqs. (2-5)) both components of the no-pair Hamiltonian are identical and the structure of the theory becomes the same as that of the nonrelativistic one. This gives the so-called spin free two-component many-electron no-pair Hamiltonian

$$
H_{+}=\sum_{i} E_{p}(i)+\sum_{i} V_{\mathrm{eff}}(i)+\sum_{i<j} \frac{1}{r_{i j}},
$$


where

$$
\begin{aligned}
& V_{\mathrm{eff}}(i)=A(i)[V(i)+\boldsymbol{R}(i) V(i) \boldsymbol{R}(i)] A(i) \\
& -\mathcal{W}(i) E_{p}(i) \mathcal{W}(i)-\frac{1}{2}\left\{(\mathcal{W}(i))^{2}, E_{p}(i)\right\}
\end{aligned}
$$

In the initial formulation of the no-pair approximation [6] the Douglas-Kroll transformation operators were constructed using the free-particle Hamiltonian only. In such a case

$$
V_{\mathrm{eff}}^{0}(i)=A(i)[V(i)+\boldsymbol{R}(i) V(i) \boldsymbol{R}(i)] A(i) .
$$

The relevance of $V$-dependent terms in the transformation procedure, already recognized by Hess [7], is also studied in this paper.

The operators $E_{p}, A$, and $\boldsymbol{R}$ may be conveniently defined in the momentum space. In order to avoid computational difficulties, the transitions between the coordinate and the momentum representations are performed within the algebraic approximation $[6,10]$. In this approximation one sets up the kinetic energy matrix $\boldsymbol{T}$ in the original orbital basis. Then one transforms $\boldsymbol{T}$ to the diagonal form and assumes that its eigenvalues are equal to $-\frac{1}{2} p^{2}$. The eigenvalues of the matrices representing $E_{p}, A$, and $\boldsymbol{R}$ are then evaluated as the appropriate functions of the eigenvalues of $\boldsymbol{T}$. Having the diagonal forms of these matrices, one transforms them to the coordinate representation using the eigenvetors of the kinetic energy matrix. Finally, by properly inserting into Eq. (8) the resolutions of the identity in the actually used basis set, the matrices representing $E_{p}$ and $V_{\text {eff }}^{0}$ are expressed as

$$
\begin{aligned}
& \left\langle a\left|E_{p}\right| b\right\rangle=\left(S C \tilde{E}_{p} C^{\dagger} S\right)_{a b}-m c^{2} S_{a b}, \\
& \left\langle a\left|V_{\mathrm{eff}}^{0}\right| b\right\rangle=\left(S C \tilde{A} C^{\dagger} V C \tilde{A} C^{\dagger} S\right)_{a b}+\left(S C \tilde{B} C^{\dagger} Y C \tilde{B} C^{\dagger} S\right)_{a b},
\end{aligned}
$$

where

$$
\begin{aligned}
& B(i)=\frac{c A(i)}{m c^{2}+E_{i}}, \\
& Y(i)=p_{i} V(i) p_{i},
\end{aligned}
$$

$S$ is the overlap matrix, tilde means that the matrix is defined in an orthogonal basis of orbitals and $C$ stands for the orthogonalization matrix. Using the algebraic approximation is the key to the success of the approaches based on the Douglas-Kroll transformation. The resulting DKH method is easily implementable within an arbitrary non-relativistic code. If the effective one-electron potential is defined according to Eq. (7), it is referred to as DKH1 method; if Eq. (8) is used instead, the method is called DKH0.

By expanding $E_{p}, A$, and $\boldsymbol{R}$ in Eqs. (8) and (9) into power series on the fine structure constant and retaining the terms up to the second order only, one obtains the MVD (also known as PCG) approximation

$$
\left(S C \tilde{E} C^{\dagger} S\right)_{a b}-m c^{2} S_{a b} \rightarrow \frac{1}{2 m}\left\langle a\left|p^{2}\right| b\right\rangle-\frac{1}{8 m^{3} c^{2}}\left\langle a\left|p^{4}\right| b\right\rangle
$$




$$
\begin{aligned}
\left(S C \tilde{A} C^{\dagger} V C \tilde{A} C^{\dagger} S\right)_{a b} & \rightarrow\langle a|V| b\rangle-\frac{1}{8 m^{2} c^{2}}\left\langle a\left|p^{2} V+V p^{2}\right| b\right\rangle \\
\left(S C \tilde{B} C^{\dagger} Y C \tilde{B} C^{\dagger} S\right)_{a b} & \rightarrow-\frac{1}{4 m^{2} c^{2}}\langle\boldsymbol{p} a|V| \boldsymbol{p} b\rangle
\end{aligned}
$$

\section{Details of the calculations}

All calculations performed in this study have been carried out with uncontracted Gaussian type orbitals (GTO's). Since this study is restricted to ground state properties, only $s$-type orbitals are required at the HF level of approximations. The initial set of $s$-type Gaussian orbital exponents has been taken from Gropen [28]. The charge distribution in the studied systems is fairly compact. Therefore the $s$-type orbital with the lowest exponent has been removed from the basis set. The remaining 18 GTO's ( $\left.\alpha_{s_{n}}^{\text {init }}\right)$ have been reoptimized with respect to the nonrelativistic HF energy by using the uniform scaling $\alpha_{s_{n}}^{\mathrm{nr}}=\alpha_{s_{n}}^{\text {init }} k_{s}^{\mathrm{nr}}$. Most of the correlation energy of two-electron ions in the $1 s^{2}$ electron configuration can be accounted for by using spd-type basis sets. For the non-relativistic and the relativistic CCSD calculations [25] in addition to the $s$-type Gaussian orbitals, five $p$-type and three $d$-type Gaussian orbitals have been used. The initial $p$-type orbital exponents have been set equal to the $s$-type orbital exponents $\alpha_{s_{n}}^{\mathrm{nr}}$ corresponding to the highest coefficients in the $1 s$ eigenvector resulting from a non-relativistic HF calculation. The final optimized $p$-type orbital exponents have been determined by the uniform scaling $\alpha_{p_{n}}^{\mathrm{nr}}=\alpha_{s_{n}}^{\mathrm{nr}} k_{p}^{\mathrm{nr}}$. Similarly, the three $d$-type exponents have been selected and optimized by the uniform scaling $\alpha_{d_{n}}^{\mathrm{nr}}=\alpha_{p_{n}}^{\mathrm{nr}} k_{d}^{\mathrm{nr}}$. The corresponding scaling factors are collected in Table I.

In the present investigation the following properties have been calculated: (i) the nonrelativistic HF energy, (ii) the first order relativistic MVD correction to the HF energy, (iii) the corrections to the HF and to the CCSD energy due to relativistic effects determined by the DKH0 method and (iv) by the DKH1 method. As reference data, the exact non-relativistic Schrödinger energies determined by Drake [29] $E^{\text {Sch }}$ and the exact numerical HF and Dirac-Hartree-Fock (DHF) energies, $E^{\mathrm{HF}}$ and $E^{\mathrm{DHF}}$, respectively, determined in this study by the GRASP program [30] have been used. The correlation energies have been obtained as differences between the CCSD and the $\mathrm{HF}$ energies or between the numerical multi-configurational (MC) and one-configurational HF or DHF energies. The numerically exact HF ionization energies, the errors in the HF ionization energies (calculated using the analytical orbitals) due to the limited orbital basis set, the exact and the CCSD electron correlation energy contributions to the ionization energies are displayed in Table II.The corrections to the ionization energies due to relativistic effects calculated by using the MVD, DKH0, and DKH1 methods are compared to the corresponding numerically exact DHF ionization energies calculated by using the GRASP program [30] in Table III. The percentage error $\delta$ displayed in the table is defined as

$$
\delta(x)=100 \frac{E^{\mathrm{DHF}}-E^{x}}{E^{\mathrm{DHF}}} \%,
$$


TABLE I

The values of scaling coefficients for $s^{-}, p^{-}$, and $d$-type atomic orbitals optimized with respect to the non-relativistic $\left(k^{\mathrm{nr}}\right)$ and the relativistic $\left(k^{\mathrm{rel}}\right)$ calculations.

\begin{tabular}{l|c|c|c|c|c|c}
\hline \hline Ions & $k_{s}^{\mathrm{nr}}$ & $k_{p}^{\mathrm{nr}}$ & $k_{d}^{\mathrm{nr}}$ & $k_{s}^{\mathrm{rel}}$ & $k_{p}^{\text {rel }}$ & $k_{d}^{\mathrm{rel}}$ \\
\hline $\mathrm{Au}^{+77}$ & 1.11 & 8.08 & 8.68 & 1.97 & 7.01 & 7.93 \\
$\mathrm{Hg}^{+78}$ & 1.13 & 7.96 & 8.71 & 2.22 & 6.28 & 7.18 \\
$\mathrm{Tl}^{+79}$ & 1.01 & 8.21 & 8.85 & 2.29 & 6.32 & 7.18 \\
$\mathrm{~Pb}^{+80}$ & 1.01 & 8.32 & 8.94 & 6.46 & 2.29 & 2.61 \\
$\mathrm{Bi}^{+81}$ & 1.02 & 8.35 & 9.03 & 6.39 & 2.37 & 2.71 \\
$\mathrm{Po}^{+82}$ & 1.03 & 8.23 & 8.91 & 7.87 & 1.93 & 2.21 \\
$\mathrm{At}^{+83}$ & 1.04 & 8.23 & 8.92 & 7.94 & 1.95 & 2.23 \\
$\mathrm{Rn}^{+84}$ & 1.02 & 9.59 & 9.15 & 20.61 & 2.26 & 2.13
\end{tabular}

TABLE II

The exact non-relativistic Hartree-Fock ionization energies $E^{\mathrm{HF}}$, the differences $\Delta E^{\mathrm{HF}}$ between $E^{\mathrm{HF}}$ and the corresponding energies obtained using the non-relativistic basis sets, the exact non-relativistic correlation energies $E_{\mathrm{nre}}^{\mathrm{corr}}$, and the correlation energies calculated using the CCSD method with the non-relativistic basis set $E_{\mathrm{nrbn}}^{\mathrm{corr}}$. The fluctuation in the last digit in $E_{\mathrm{nrbn}}^{\mathrm{corr}}$ are due to the finite basis set effects. All results are in mhartrees.

\begin{tabular}{c|c|c|c|c}
\hline \hline Ions & $E^{\mathrm{HF}}$ & $\Delta E^{\mathrm{HF}}$ & $E_{\text {nre }}^{\text {corr }}$ & $E_{\text {nrbn }}^{\text {corr }}$ \\
\hline $\mathrm{Au}^{+77}$ & 3071237 & 7 & -46 & -44 \\
$\mathrm{Hg}^{+78}$ & 3150112 & 8 & -46 & -45 \\
$\mathrm{Tl}^{+79}$ & 3229987 & 8 & -46 & -45 \\
$\mathrm{~Pb}^{+80}$ & 3310862 & 8 & -46 & -44 \\
$\mathrm{Bi}^{+81}$ & 3392737 & 8 & -46 & -44 \\
$\mathrm{Po}^{+82}$ & 3475612 & 8 & -46 & -44 \\
$\mathrm{At}^{+83}$ & 3559487 & 9 & -46 & -45 \\
$\mathrm{Rn}^{+84}$ & 3644362 & 9 & -46 & -45
\end{tabular}

where $E^{\mathrm{DHF}}$ and $E^{x}$ denote, respectively, the relativistic corrections to the exact DHF energy and to the energy in question. The DKH1 energies have been calculated using basis sets optimized both at the non-relativistic and at the relativistic level of approximation. Finally, the electron correlation energies calculated on the relativistic level of approximation are displayed in Table IV. 
TABLE III

DHF relativistic corrections to ionization energies $\left(E_{\mathrm{rel}}^{\mathrm{DHF}}\right)$ and the percentage errors given by the method specified. Subscripts nr and rel refer to using the non-relativistic and relativistic basis set, respectively. All results are in mhartrees.

\begin{tabular}{c|c|c|c|c|c}
\hline \hline Ions & $E_{\mathrm{rel}}^{\mathrm{DHF}}$ & $\delta\left(\mathrm{MVD}_{\mathrm{nr}}\right)$ & $\delta\left(\mathrm{DKH}_{\mathrm{nr}}\right)$ & $\delta\left(\mathrm{DKH} 1_{\mathrm{nr}}\right)$ & $\delta\left(\mathrm{DKH} 1_{\mathrm{rel}}\right)$ \\
\hline $\mathrm{Au}^{+77}$ & 306394 & 18.39 & -44.17 & 4.07 & 4.04 \\
$\mathrm{Hg}^{+78}$ & 324143 & 18.87 & -44.50 & 4.11 & 4.07 \\
$\mathrm{Tl}^{+79}$ & 342746 & 19.33 & -44.80 & 4.14 & 4.10 \\
$\mathrm{~Pb}^{+80}$ & 362240 & 19.63 & -45.11 & 4.18 & 4.13 \\
$\mathrm{Bi}^{+81}$ & 382664 & 20.27 & -45.43 & 4.22 & 4.16 \\
$\mathrm{Po}^{+82}$ & 404057 & 20.76 & -45.76 & 4.26 & 4.19 \\
$\mathrm{At}^{+83}$ & 426460 & 21.25 & -46.09 & 4.29 & 4.21 \\
$\mathrm{Rn}^{+84}$ & 449919 & 21.75 & -46.41 & 4.34 & 4.22
\end{tabular}

\section{TABLE IV}

Non-relativistic correlation energies $\left(E_{\mathrm{num}}^{\mathrm{corr}}\right)$ and the relativistic corrections $\varepsilon_{\text {num }}$ calculated by the numerical MCHF and MCDHF methods for $(1 s+2 s+2 p+$ $3 d)^{2}$ configuration compared with the corresponding quantities ( $E_{\mathrm{rlbn}}^{\mathrm{corr}}$ and $\varepsilon_{\mathrm{rlbr}}$ ) calculated by CCSD and DKH1 methods using the relativistic orbital basis set defined in the text. All results are in mhartrees.

\begin{tabular}{c|c|c|c|c}
\hline \hline Ions & $E_{\text {num }}^{\text {corr }}$ & $\varepsilon_{\text {num }}$ & $E_{\text {rlbn }}^{\text {corr }}$ & $\varepsilon_{\text {rlbr }}$ \\
\hline $\mathrm{Au}^{+77}$ & -39.7 & -2.95 & -44.4 & -33.4 \\
$\mathrm{Hg}^{+78}$ & -39.7 & -3.14 & -44.5 & -34.6 \\
$\mathrm{Tl}^{+79}$ & -39.7 & -3.34 & -44.5 & -35.8 \\
$\mathrm{~Pb}^{+80}$ & -39.7 & -3.55 & -44.5 & -37.2 \\
$\mathrm{Bi}^{+81}$ & -39.7 & -3.77 & -44.5 & -38.5 \\
$\mathrm{Po}^{+82}$ & -39.7 & -4.00 & -44.5 & -39.9 \\
$\mathrm{At}^{+83}$ & -39.7 & -4.25 & -44.5 & -41.3 \\
$\mathrm{Rn}^{+84}$ & -39.7 & -4.51 & -44.5 & -42.9
\end{tabular}

\section{Results and discussion}

According to the data of Table III both the MVD and the DKH0 methods fail to account correctly for the contribution to the ionization energy of the two-electron heavy atomic ions due to relativistic effects. By using these approximations at most the order of magnitude of the relativistic correction to the ion- 
ization energies can be estimated. The performance of both methods may be interpreted by analyzing $Z / c$-expansions of the corresponding energies evaluated for hydrogen-like atoms [31, 32]. In the case of the MVD method, only terms up to $Z^{4} / c^{2}$ are taken into account. The first term of the expansion which is neglected in this approach, is equal (in mhartree) to

$$
\Delta^{\mathrm{H}}=62.5 \frac{\mathrm{Z}^{6}}{\mathrm{c}^{4}}
$$

and leads to an increase in the relativistic correction [31]. In the present calculation the relativistic corrections are underestimated by the MVD approximation. The least-square fit shows that the corresponding absolute error, as a function of $Z$, behaves as

$$
\Delta^{\mathrm{He}}=82 \frac{\mathrm{Z}^{6}}{\mathrm{c}^{4}}
$$

The difference between $\Delta^{\mathrm{H}}$ and $\Delta^{\mathrm{He}}$ is a consequence of the non-hydrogenic character of the wave function and of an effective influence of the higher-order terms.

In the case of the DKH0 method, using the free-particle projector introduces admixtures of negative-energy solutions which pollute the positive energy state [32]. In the corresponding $Z$-expansion of the energy of a hydrogen-like atom, this results in the presence of a spurious term proportional to $Z^{5}$. As one can check by applying the least-squares procedure to the data displayed in Table III, the absolute error of the DKH0 method scales with $Z^{5}$. Then, the errors of the MVD and of the DKH0 methods, which in the case under consideration amount, respectively, to $20 \%$ and $45 \%$ of the total relativistic correction, are due to the high-order terms either not included (in the MVD Hamiltonian) or are incorrectly represented (in the DKH0 Hamiltonian). Since the leading term of the total relativistic correction is proportional to $Z^{4}$, the percentage errors of the MVD and of the DKH0 methods have to be, approximately, proportional to $Z^{2}$ and to $Z$, respectively. This behaviour of the errors may also be seen from Table III. One should remember $[31,32]$ that in the case of the MVD method, the next missing term in the $Z$-expansion of the energy is proportional to $Z^{8}$, while in the case of the DKH0 method, the next incorrectly represented term is proportional to $Z^{6} \log Z$. Therefore the departure of $\delta$ (MVD) from the $Z^{2}$ behaviour is considerably smaller than the departure of $\delta$ (DKH 0 ) from the linearity in $Z$ (as it can be seen from the data in Table III).

The values of the relativistic corrections resulting from the DKH1 method are correct up to $4 \%$ (the error increases with $Z$ from $4.04 \%$ for $\mathrm{Au}^{+77}$ to $4.22 \%$ for $\mathrm{Rn}^{+84}$ ). Reoptimizing the basis sets by using the DKH1 Hamiltonian improves the corrections due to relativistic effects only slightly. The remaining differences between the DHF and DKH1 results may be attributed to the approximate form of the no-pair Hamiltonian and to the use of the algebraic approximation (this contribution to the error is expected to be $Z$-independent however it depends upon the quality of the orbital basis set).

The $4 \%$ error of the DKH1 relativistic correction corresponds to the absolute error ranging from 12 hartree for $\mathrm{Au}^{+77}$ to 19 hartree for $\mathrm{Rn}^{+84}$. This error is more than two orders of magnitude larger than the absolute value of the electron 
correlation energy, which in this case does not exceed 0.05 hartree. Therefore one should not expect that for the systems considered in this paper relativistic corrections to the electron correlation energy may be well described by the DKH method. However, the major source of the error, i.e. the algebraic approximation, influences the one-electron integrals only and, consequently, modifies both non-correlated and correlated energies in a similar way. Therefore some considerable compensations of the errors may be expected. The degree of this compensation, to a certain extent, determines the limits of applicability of the DKH method.

The relativistic correction to the electron correlation energy, defined as the difference between correlation energies derived from a relativistic and from a non-relativistic Hamiltonian

$$
\varepsilon=\left(E^{\mathrm{DC}}-E^{\mathrm{DHF}}\right)-\left(E^{\mathrm{Sch}}-E^{\mathrm{HF}}\right)
$$

(where $E^{\mathrm{DC}}$ denotes the appropriate eigenvalue of the Dirac-Coulomb Hamiltonian) is referred to as the Coulomb part of the correlation-relativity cross-term energy [33-35]. The second part of the relativistic correction to the electron correlation energy is due to the Breit operator and is not considered in this paper. The lowest-order term in $Z / c$ expansion of $\varepsilon$ vanishes $[33,35]$. Therefore, except for a large $Z$, the values of $\varepsilon$ are very small. In particular, for the ground states of the helium-like ions with $Z<60$ they do not exceed 0.5 mhartree [34]. The non-relativistic correlation energies and the corresponding relativistic corrections $\varepsilon_{\text {num }}$, calculated using the numerical MCDF procedure with $(1 s+2 s+2 p+3 d)^{2}$ configurations included, as well as the corresponding values $\varepsilon_{\text {rlbr }}$ derived from the CCSD, HF, and DKH1 methods are collected in Table IV. As one can see, the non-relativistic correlation energies obtained by the CCSD method and by the numerical MCHF procedure are in fairly good agreement (the differences are mainly due to the limited set of configurations included in the numerical MCHF calculations). On the other hand, as expected, the relativistic DKH1-CCSD values of $\varepsilon_{\text {rlbr }}$ are completely wrong, though the degree of compensation of the error $\delta(\mathrm{DKH} 1)$ is surprisingly large. The origin of the non-compensated error may be deduced from the type of its $Z$-dependence. The least square fit of the correction derived from the numerical MCDHF calculations gives

$$
\varepsilon_{\text {num }} \approx-46\left(\frac{Z}{c}\right)^{5}
$$

and in the case of the DKH1-CCSD correction,

$$
\varepsilon_{\mathrm{rlbr}} \approx-174\left(\frac{Z}{c}\right)^{3} \text {. }
$$

Then, the values of $\varepsilon_{\text {rlbr }}$ do not describe the Coulomb part of the correlation-relativity cross-terms, which behave as $Z^{5}$, but are dominated by the residual values of $\delta(\mathrm{DKH} 1)$ being proportional to $Z^{3}$.

\section{Conclusions}

According to the results of the present investigation the contribution to the ionization energy of the two-electron heavy atomic ions due to relativistic effects 
is well represented by the Hamiltonian resulting from the Douglas-Kroll-Hess transformation taking into account the nuclear potential (DKH1). In the present case the corrections resulting from the DKH1 approximation are correct up to $4 \%$. Both the MVD and the DKH0 approximations fail to account correctly for these corrections.

\section{Acknowledgments}

This work has been supported by the Committee for Scientific Research under the research grant No. 3 T09A 11617.

\section{References}

[1] O. Visser, L. Visscher, P.J.C. Aerts, W.C. Nieuwpoort, Theor. Chim. Acta 81, 405 (1992); L. Visscher, T. Saue, W.C. Nieuwpoort, K. Fægri, O. Gropen, J. Chem. Phys. 99, 6704 (1993).

[2] K. Dyall, J. Chem. Phys. 98, 9678 (1993).

[3] L. Visser, Relativity and Electron Correlation in Chemistry, Rijksuniversiteit Groningen Press, Groningen 1993 and references therein.

[4] J. Sucher, Phys. Rev. A 22, 348 (1980).

[5] M. Douglas, N.M. Kroll, Ann. Phys. 82, 89 (1974).

[6] B.A. Hess, Phys. Rev. A 32, 756 (1985).

[7] B. A. Hess, Phys. Rev. A 33, 3742 (1986).

[8] J. Almlöf, K. Fægri, H.H. Grelland, Chem. Phys. Lett. 114, 53 (1985).

[9] M. Barysz, A.J. Sadlej, J.G. Snijders, Int. J. Quantum Chem. 65, 225 (1997).

[10] R. Samzow, B.A. Hess, G. Jansen, J. Chem. Phys. 96, 1227 (1992).

[11] B.A. Hess, P. Chandra, Phys. Scr. 36, 412 (1987).

[12] U. Kaldor, B.A. Hess, Chem. Phys. Lett. 230, 1 (1994).

[13] M.M. Gleichmann, B.A. Hess, J. Chem. Phys, 101, 9691 (1994).

[14] C.L. Collins, K.G. Dyall, H.G. Schaefer III, J. Chem. Phys. 102, 2024 (1995).

[15] M. Barysz, M. Urban, Adv. Quant. Chem. 28, 21 (1997).

[16] M. Barysz, P. Pyykkö, Chem. Phys. Lett. 285, 398 (1998).

[17] R.D. Cowan, D.C. Griffin, J. Opt. Soc. Am. 66, 1010 (1976).

[18] J.D. Morrison, R.E. Moss, Mol. Phys. 41, 491 (1980); W. Kutzelnigg, Z. Phys. D 11, 15 (1989).

[19] C. Park, J.E. Almlöf, Chem. Phys. Lett. 231, 269 (1994).

[20] J.P. Desclaux, K.T. Cheng, Y.K. Kim, J. Phys. B 12, 3819 (1979); I.P. Grant, B.J. McKenzie, J. Phys. B 13, 2671 (1980).

[21] B.P. Das, I.P. Grant, J. Phys. B 19, L7 (1986); J. Hata, I.P. Grant, J. Phys. B 16, 507, 523 (1983); A. Ynnerman, C. Froese-Fischer, Phys. Rev. A 51, 2020 (1995).

[22] P. Chandra, B.A. Hess, Theor. Chim. Acta 88, 183 (1994).

[23] M.M. Gleichmann, B.A. Hess, Chem. Phys. Lett. 227, 229 (1994). 
[24] A. Pizlo, G. Jansen, B.A. Hess, W. von Niessen, J. Chem. Phys. 98, 3945 (1993).

[25] M. Kolaski, S. Kucharski, The nonrelativistic CCSD program has been adapted to the Open Mol system and has been used in the present calculations.

[26] G.H.F. Diercksen, G.G. Hall, Comput. Phys. 8, 215 (1994).

[27] M. Barysz, W. Duch, N. Flocke, P. Graf, N.E. Grüner, J. Karwowski, V. Kellö, L. Laaksonen, E.S. Schreiner, N.S. Scott, M. Urban, G.H.F. Diercksen, OpenMol System of Quantum Chemistry Programs (1995) has been used in our calculations.

[28] O. Gropen, J. Comp. Chem. 8, 982 (1987).

[29] G.W. Drake, Can. J. Phys. 66, 586 (1988).

[30] I.P. Grant, B.J. McKenzie, P.H. Norrington, D.F. Mayers, N.C. Pyper, Comput. Phys. Commun. 21, 207 (1980); K.G. Dyall, I.P. Grant, C.T. Johnson, F.A. Parpia, E.P. Plummer, Comput. Phys. Commun. 55, 425 (1989).

[31] H.A. Bethe, E.E. Salpeter, Quantum Mechanics of One- and Two-Electron Systems, Handbuch der Physik, Vol. 35, Springer Verlag, Berlin 1957.

[32] J.L. Heully, I. Lindgren, E. Lindroth, A.M. Martensson-Pendrill, Phys. Rev. A 33, 4426 (1986); W. Kutzelnigg, Phys. Scri. 36, 416 (1987); H.M. Quiney, in: Relativistic Effects in Atoms and Molecules, in series: Methods in Computational Chemistry, Vol. 2, Ed. S. Wilson, Plenum Press, New York 1988, p. 227.

[33] A.M. Ermolaev, M. Jones, J. Phys. B 6, 1 (1973).

[34] O. Gorceix, P. Indelicato, J.P. Desclaux, J. Phys. B 20, 639 (1987); J. Styszyński, J. Karwowski, J. Phys. B 21, 2389 (1988); F.A. Parpia, I.P. Grant, J. Phys. B 23, 211 (1990).

[35] J. Karwowski, J. Styszyński, W.H.E. Schwarz, J. Phys. B 24, 4877 (1991); Erratum: 25, 2763 (1992). 